\title{
Reply to "Comment on 'Slowness-azimuth corrections of teleseismic events for IMS primary arrays in China' by Chunyue Hao and Zhong Zheng”' by Lei Li
}

\author{
Chunyue Hao $\cdot$ Zhong Zheng
}

Received: 14 March 2011 / Accepted: 28 March 2011 / Published online: 26 April 2011

(C) Springer Science+Business Media B.V. 2011

Small aperture seismic array has low resolution of slowness and back-azimuth. Some small seismic arrays have 25 elements, such as Norwegian Seismic Array (NORESS), Arctic Regional Seismic Array (ACRESS), German Experimental Seismic Array System (GERESS), and others, and some array have 16-25 elements, such as Matsushiro Array (in Japan) (MJAR) and Hagfors array (in Sweden) (HFS). The Lanzhou array (in China) (LZDM), which has nine elements, is also one of these kinds of small aperture arrays, which might be affected by very high deviations of back-azimuth and slowness. Hence, a seismic array needs a series of corrections, like altitude and ellipticity correction, Source-Specific Station Correction, slowness-azimuth correction, struc- ture correction, and so on. The aim of these corrections is to enhance the accuracy of the observation values. The improvement of the location accuracy is a challenge in seismology, up to now. When you apply a kind of correction to an array, it may improve the location to some extent, but it cannot eliminate all the errors.

Since every kind of correction can improve the observation values and cannot achieve theoretical ones, the slowness-azimuth correction to the LZDM is only one of these processes; hence, the result is only a result without altitude correction, ellipticity correction, structure correction, and so on.

The altitude correction of LZDM may be our next work.
C. Hao $(\varangle) \cdot$ Z. Zheng Institute of Geophysics, CEA, Beijing, China e-mail: haochunyue@gmail.com 